\title{
Hypertension Enhances Advanced Atherosclerosis and Induces Cardiac Death in Watanabe Heritable Hyperlipidemic Rabbits
}

Bo Ning, ${ }^{\dagger}$ Y Yajie Chen, ${ }^{\dagger}$ Ahmed Bilal Waqar, ${ }^{\dagger}$ Haizhao Yan, ${ }^{\dagger}$ Masashi Shiomi, ${ }^{\ddagger}$ Jifeng Zhang, ${ }^{\S}$ Y. Eugene Chen, ${ }^{\S}$ Yanli Wang, ${ }^{*}$ Hiroyuki Itabe, " Jingyan Liang, $\|$ and Jianglin Fan ${ }^{\dagger}$

\begin{abstract}
From the College of Clinical Medicine and Department of Pathology, ${ }^{*}{ }_{i}{ }^{\prime}$ an Medical University, Xi'an, China; the Department of Molecular Pathology, ${ }^{\dagger}$ Faculty of Medicine, Graduate School of Medical Sciences, University of Yamanashi, Yamanashi, Japan; the Institute for Experimental Animals, ${ }^{\ddagger}$ Kobe University School of Medicine, Kobe, Japan; the Center for Advanced Models for Translational Sciences and Therapeutics, ${ }^{\S}$ University of Michigan Medical Center, Ann Arbor, Michigan; the Division of Biological Chemistry, "Department of Molecular Biology, Showa University, School of Pharmacy, Tokyo, Japan; and the Research Center for Vascular Biology," School of Medicine, Yangzhou University, Yangzhou, China
\end{abstract}

Accepted for publication

August 6, 2018

Address correspondence to Jianglin Fan, M.D., Ph.D., Department of Molecular Pathology, University of Yamanashi, 1110 Shimokato, Yamanashi 409-3898, Japan. E-mail: jianglin@ yamanashi.ac.jp.

\begin{abstract}
Hypertension is a major risk factor for the development of atherosclerosis. Cardiovascular risk has been reported to be significantly increased in hyperlipidemic patients with hypertension. However, it is not clear whether hypertension can directly destabilize plaques, thereby enhancing cardiovascular events. To examine whether hypertension enhances the development of atherosclerosis and increases plaque vulnerability, we generated hypertensive Watanabe heritable hyperlipidemic (WHHL) rabbits by surgical removal of one kidney and partial ligation of the other renal artery and compared the nature of aortic and coronary atherosclerosis in hypertensive WHHL rabbits with normotensive WHHL rabbits. All hypertensive WHHL rabbits died from 34 to 56 weeks after surgery, whereas no normotensive WHHL rabbits died. Pathologic examinations revealed that hypertensive WHHL rabbits showed different degrees of myocardial infarction caused by severe coronary stenosis along with myocardial hypertrophy. Furthermore, aortic lesions in hypertensive WHHL rabbits exhibited a higher frequency of intraplaque hemorrhage and vulnerable plaques than those in normotensive WHHL rabbits. These results indicate that hypertension induced by the surgical removal of one kidney and partial ligation of the other renal artery method in WHHL rabbits may not only enhance the development of atherosclerosis but also destabilize the plaques, increasing cardiac death. (Am J Pathol 2018, 188: 2936-2947; https://doi.org/10.1016/j.ajpath.2018.08.007)
\end{abstract}

Hypertension has long been known to increase the prevalence of coronary heart disease ${ }^{1}$ and also to increase the extent and severity of atherosclerosis in both humans ${ }^{1,2}$ and experimental animals. ${ }^{3-5}$ However, treatment with antihypertensive drugs significantly reduces the development of atherosclerosis and cardiovascular events. ${ }^{6}$ Hypertension may accentuate the progression of atherosclerosis through several mechanisms, including induction of mechanical injury to endothelial cells, enhancement of low-density lipoprotein (LDL) permeability to the intima, and adherence of monocytes to endothelial cells. ${ }^{7}$ Elevated plasma levels of angiotensin II, which is often present in hypertensive (HTN) subjects, enhance atherosclerosis and the formation of aortic aneurysms in hyperlipidemic mice. ${ }^{8,9}$ In addition,
Tsukuba HTN mice, which transgenically express both human angiotensinogen and renin genes, exhibited extensive atherosclerosis in the aortic root when challenged with a high-fat diet. ${ }^{10}$

\footnotetext{
Supported in part by the National Key Research and Development Program of China grant 2016YFE0126000 (J.L. and J.F.), the National Natural Science Foundation of China grants 81570392 (J.L.) and 81770457 (J.F.), JSPS KAKENHI grant 15H04718 (J.F.), JSPS-CAS under the JapanChina Research Cooperative Program (J.F.), the Natural Science Foundation of Shaanxi Province grant 2017JZ028 (J.F.), and NIH grants R01HL117491 and R01HL129778 (Y.E.C.).
}

B.N., Y.C., and A.B.W. contributed equally to this work.

Disclosures: None declared. 
Hypertension primarily affects small arteries and arterioles (diameter $<200 \mu \mathrm{m}$ ) and causes hyaline arteriolosclerosis. Indeed, hypertension alone in the absence of elevation of plasma cholesterol levels usually cannot induce the atherosclerosis commonly observed in large arteries, ${ }^{11}$ suggesting that hypercholesterolemia is essential for the initiation and formation of atherosclerosis, whereas hypertension is an enhancer for its progression. However, it is not clear whether hypertension affects atherosclerotic complications such as myocardial infarction (MI).

Watanabe heritable hyperlipidemic (WHHL) rabbits are a unique model for the study of human hypercholesterolemia and atherosclerosis because they develop spontaneous hypercholesterolemia and atherosclerosis because of deficient LDL receptor functions. ${ }^{12,13}$ Chobanian et $\mathrm{al}^{5}$ reported that when WHHL rabbits were subjected to a short period of hypertension, they developed greater aortic atherosclerosis than normotensive (NTN)-WHHL rabbits, ${ }^{14}$ whereas this enhancement of atherosclerosis can be alleviated by treatment with captopril, an angiotensin-conversing enzyme inhibitor. ${ }^{15}$ Infusion of angiotensin II into WHHL rabbits by osmotic minipumps destabilizes the coronary plaques and causes MI. ${ }^{16}$ Although the short-term effects of blood pressure elevation on the development of atherosclerosis have been extensively studied in different experimental animals, it has not been confirmed whether long-term blood pressure elevation affects the nature of atherosclerosis, coronary atherosclerosis, or cardiac pathologic processes. This is an important issue because hypertension is a lifelong condition in human patients if not properly treated. Because most animal models cannot sustain to the long period of cholesterol diet feeding and rarely develop coronary atherosclerosis or MI, this hypothesis has been difficult to test by using experimental animal models. It has been reported that WHHL rabbits are susceptible to coronary atherosclerosis and MI at an old age. ${ }^{13}$ Therefore, we examined whether a prolonged period of hypertension affects coronary atherosclerosis or subsequent MI in WHHL rabbits without a cholesterol-rich diet. The aim of this study was to characterize and compare the nature of aortic and coronary atherosclerotic lesions in HTN- and NTN-WHHL rabbits and to evaluate changes in the myocardium possibly related to ischemia or hypertension. We were particularly interested in whether long-term hypertension has any effects on plaque stability and cardiac-related events. Our present study demonstrated that prolonged blood pressure elevation not only enhances advanced atherosclerosis but also induces cardiac death in WHHL rabbits.

\section{Materials and Methods}

\section{Animals}

Male WHHL rabbits aged 8 months in which aortic and coronary atherosclerosis was well developed were used. ${ }^{13}$ To investigate the influence of hypertension on the development of atherosclerosis, hypertension was induced by surgical removal of one kidney and partial ligation of the other renal artery (1K1C), ${ }^{5}$ and the NTN group underwent sham surgery as a control. In brief, rabbits were anesthetized with ketamine/ medetomidine and placed in dorsal recumbence. The abdominal hair was then shaved, and the abdomen was sterilized with betadine. After opening the abdominal cavity, the left kidney was removed with the adrenal gland remaining, and the right renal artery was partially ligated by using a silver restriction clip with a gap of $0.508 \mathrm{~mm} .{ }^{5}$ Rabbits were fed a standard chow diet (CLEA Japan Inc., Tokyo, Japan) throughout the experiment. Animal experiments were performed after approval from the Animal Care Committee of the University of Yamanashi and conformed to NIH's Guide for the Care and Use of Laboratory Animals. ${ }^{17}$

\section{Measurement of BP and Plasma Variables}

The blood pressure (BP) and heart rate were examined every month during the experiment as described previously. ${ }^{16,18}$ In brief, to avoid stress from the environment, rabbits were placed in a quiet room at least 30 minutes before BP measurement. The medial auricular artery was cannulated with a 23-gauge cannula, and BP and heart rate were recorded with a transducer. These data were collected from each rabbit for approximately 15 to 20 minutes by BP amplifier (AD Instruments, Tokyo, Japan). BP was calculated with Chart5 Pro software version 5.5 (AD Instruments).

Blood was collected from rabbits after 16-hour fasting. Plasma levels of total cholesterol, triglycerides, and highdensity lipoprotein cholesterol were measured biweekly by using Wako assay kits (Wako Pure Chemical Industries, Osaka, Japan). ${ }^{19}$ To evaluate the renal functions, plasma creatinine and urea were measured with a commercially available enzymatic assay kit (Cat-KGE005; R\&D System Inc., Minneapolis, MN) and urea assay kit (DIUR-100) (BioAssay Systems, Hayward, CA), respectively. In addition, angiotensin II levels in plasma were determined with an enzyme-linked immunosorbent assay kit (Enzo Life Sciences, Inc., New York, NY) according to the manufacturer's instructions.

\section{Pathologic Examinations}

At 56 weeks after surgery, all WHHL rabbits were sacrificed by an overdose injection of sodium pentobarbital $(100 \mathrm{mg} /$ $\mathrm{kg}$ ). Aortic trees were isolated, and periaortic adipose tissue was removed. Aortas were opened and fixed on a corkboard with $10 \%$ neutral-buffered formalin solution. The hearts and brains from each animal were weighed, photographed, and then fixed in $10 \%$ formalin. After fixing, whole aortic trees were stained with Sudan IV and analyzed as described previously. ${ }^{20}$ For microscopic quantification of the aortic lesions, the aorta was divided into three parts: aortic arch and thoracic and abdominal aortas. ${ }^{20}$ Each part was further cut into several segments ( 8 for the arch, 20 for the thoracic aorta, and 30 for the abdominal aorta) and embedded in paraffin. 
Table 1 Monoclonal Antibodies Used for Immunohistochemical Staining

\begin{tabular}{llll}
\hline Antibodies & Dilution & Catalog number & Manufacturer \\
\hline Macrophage (RAM) & $1: 400$ & M0633 & Dako Co., Carpinteria, CA \\
Muscle actin (HHF35) & $1: 300$ & M0635 & Dako Co. \\
MDA-oxidized LDL & $1: 400$ & Ab63975 & Abcam plc., Cambridge, UK \\
Copper-oxidized LDL & $1: 400$ & 020418 & Division of Biological Chemistry, Showa University, Japan \\
\hline
\end{tabular}

LDL, low-density lipoprotein; MDA, malondialdehyde.

Specimens were cut in 3- $\mu \mathrm{m}$ serial sections and used for hematoxylin and eosin, elastic van Gieson, and Masson's trichrome staining. Immunohistochemical staining was performed by using monoclonal antibodies against rabbit macrophage RAM11 (Dako Co., Carpinteria, CA) and smooth muscle $\alpha$-actin (Dako Co.). ${ }^{21}$ In addition, the lesion types were quantified as follows: fatty streaks (type II lesions: mainly composed of foam cells), fibrotic lesions [type III lesions: mainly composed of smooth muscle cells (SMCs) and extracellular matrix], and complicated or advanced lesions (type IV or V fibrous plaques combined with lipid/ necrotic core or with calcification) according to the American Heart Association classification. ${ }^{22}$ The lengths of different lesions on each specimen were measured and averaged. ${ }^{21}$ HTN-WHHL rabbits that died at night during the experiment were autopsied in the morning. Autopsy included gross and microscopic examinations such as body appearance examination, thoracic and abdominal cavity examination, and all important organs (liver, adrenal, spleen, kidneys, stomach and intestines, heart and lung, and brain). After autopsy, all organs collected were fixed in $10 \%$ neutral-buffered formalin solution, and specimens were routinely stained with hematoxylin and eosin for light microscopy examinations. In the aortas of HTN-WHHL rabbits, many aortic lesions that contained hemorrhagic sites that varied in size from approximately $3 \mu \mathrm{m}$ were observed (Analysis of Heart and Coronary Atherosclerosis). To quantify these hemorrhagic lesions, the hemorrhagic number was calculated and plotted on the sections by using hematoxylin and eosin-stained specimens under a light microscope. ${ }^{23}$ In addition, immunohistochemical staining of these hemorrhagic areas was performed with two monoclonal antibodies against either copper-oxidized LDL (a gift from Dr. Hiroyuki Itabe, Showa University, Tokyo, Japan) or malondialdehyde-oxidized LDL (Ab63975; Abcam plc., Cambridge, UK) (Table 1).

\section{Analysis of Heart and Coronary Atherosclerosis}

The heart appearance was first examined and the weight was measured. The hearts were then dissected by using the methods for analysis of coronary atherosclerosis as described previously. ${ }^{16,24}$ In brief, the hearts were dissected into five blocks according to the distribution of coronary arteries. Blocks I and II contained the main trunks of the left and right coronary arteries, whereas blocks III to V contained the small branches of the left circumflex, left descending artery, and obtuse marginal arteries along with arterioles with a diameter $<200 \mu \mathrm{m}$. The stenosis rate $(\%)$ was defined as atherosclerotic lesions area/lumen area. Block $\mathrm{V}$ included the maximal vertical surface of both right and left ventricles and was thus used for histologic and morphometric analyses of the myocardium, including MI, cardiac fibrosis, and myocardial

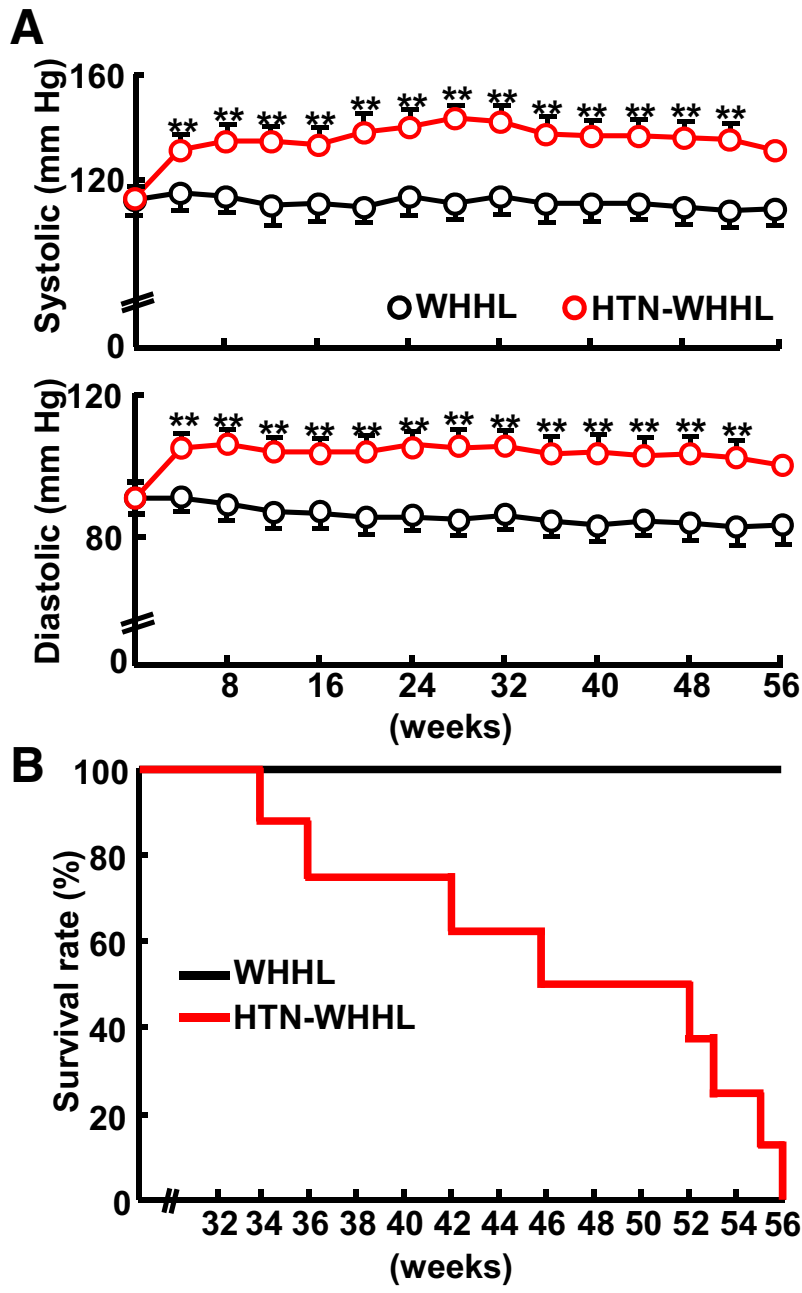

Figure 1 Analysis of blood pressure and survival rate of Watanabe heritable hyperlipidemic (WHHL) rabbits. A: Systolic and diastolic pressure was measured monthly after renovascular hypertension. B: Kaplan-Meier analysis of cumulative rates of survival among WHHL rabbits; $P=0.002$ versus normotensive WHHL rabbit. Data are expressed as means \pm SEM. $n=7$ normotensive WHHL rabbits; $n=8$ hypertensive (HTN) Watanabe heritable hyperlipidemic (WHHL) rabbits. ${ }^{*} P<0.01$ versus normotensive WHHL rabbits. 
A
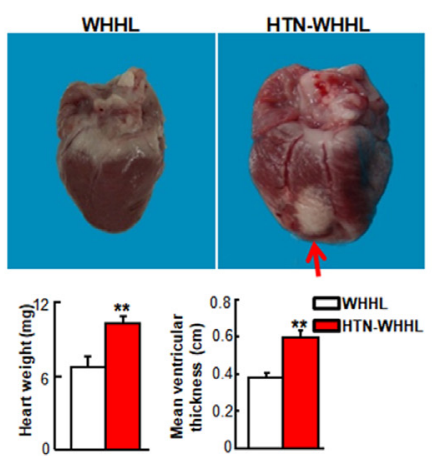

C
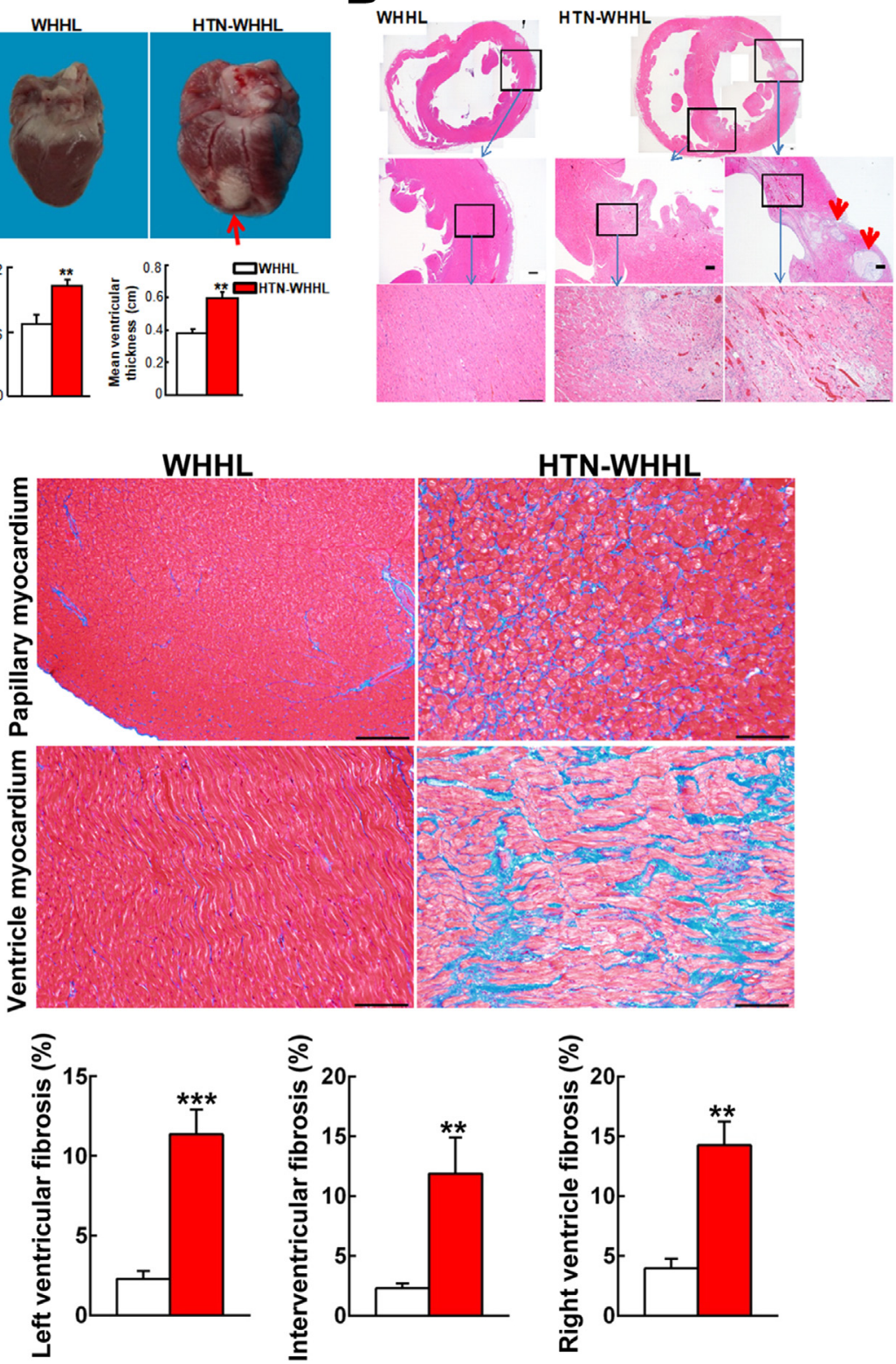

D

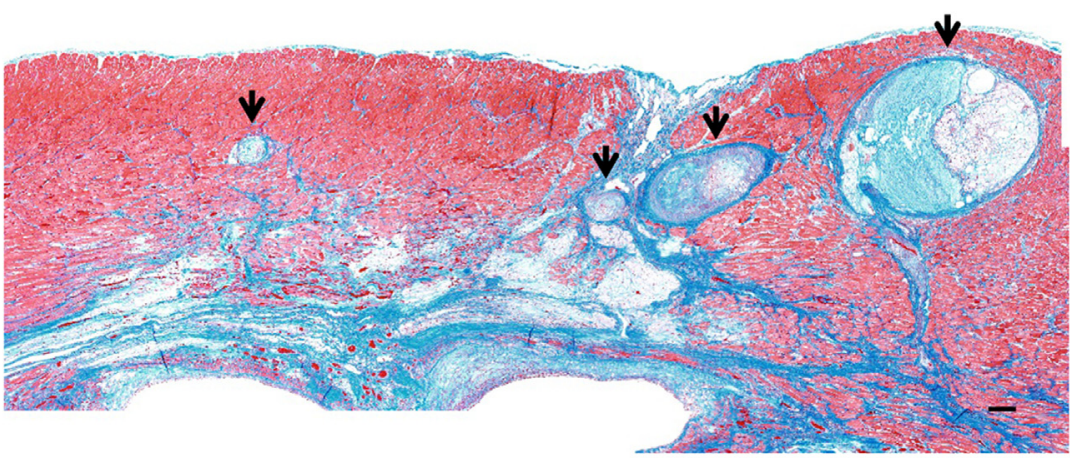

Figure 2 Pathologic analysis of the heart. A: Grossly, hearts of hypertensive (HTN) Watanabe heritable hyperlipidemic (WHHL) rabbits were larger in size and heavier with a thickened ventricular wall than those of normotensive WHHL rabbits. On the surface of the heart of HTN-WHHL rabbits, a whitish area (chronic myocardial infarction), indicated by a red arrow, can be seen. B: Histologic features of the heart. Specimens were stained with hematoxylin and eosin (HE), and chronic myocardial infarction can be seen in the left ventricle and interventricular septa of HTNWHHL rabbits (top right panel). Myocardial infarction lesions were characterized by marked fibrosis in which cardiomyocyte eosinophilic degeneration, hemorrhage, angiogenesis, and inflammatory cell infiltration were contained. Occlusive atherosclerotic lesions within myocardium are included (red arrowheads). Boxed areas are shown at higher magnification below. $\mathbf{C}$ and $\mathbf{D}$ : Masson trichrome (MT) staining revealed that nonmyocardial infarction (C) area and myocardial infarction (D) was associated with marked fibrosis, stained in blue. The specimens of papillary myocardium and the left ventricle were stained with MT (C, top and middle rows), and fibrosis was calculated by measuring the MT staining area (C, bottom row). Four coronary arteries/arterioles show prominent occlusive features (black arrowheads). Data are expressed as means \pm SEM. $n=7$ normotensive WHHL rabbits; $n=8$ HTNWHHL rabbits. ${ }^{* *} P<0.01,{ }^{* * *} P<0.001$ versus normotensive WHHL rabbits. Scale bars $=100 \mu \mathrm{m}$. hypertrophy. The thickness of the left ventricular wall and interventricular septum was measured and averaged. Cardiac fibrosis defined by Masson's trichrome staining was calculated by using an image analysis system.

\section{Statistical Analysis}

All values were expressed as means \pm SEM. The KaplanMeier estimator and the log-rank test were used to evaluate 

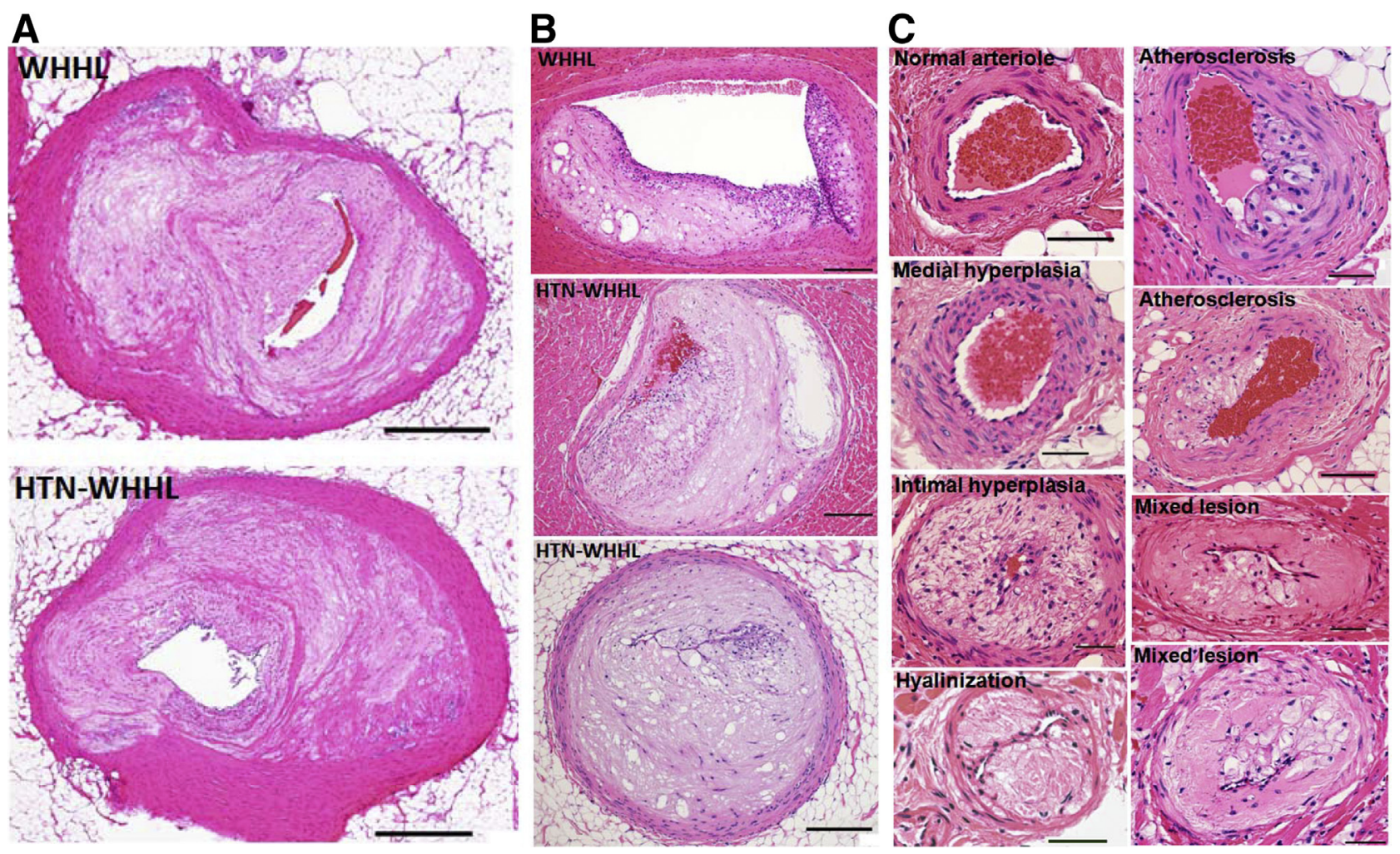

Figure 3 Representative micrographs of coronary atherosclerosis in Watanabe heritable hyperlipidemic (WHHL) and hypertensive (HTN)-WHHL rabbits. Epicardial coronary arteries exhibited prominent atherosclerosis in both groups (A), but small arteries (B) and arterioles (C) of HTN-WHHL rabbits showed various changes, including extensive stenosis (B), medial and intimal hyperplasia, hyalinization, fatty streaks, and mixed lesions that contained both hyalinization and fatty streaks (C). A normal arteriole was from a normotensive WHHL rabbit, and all other lesions were from HTN-WHHL rabbits (C). All specimens were stained with hematoxylin and eosin. Scale bars: $500 \mu \mathrm{m}$ (A); $200 \mu \mathrm{m}$ (B); $50 \mu \mathrm{m}$ (C).

the survival rate after surgery-induced hypertension in WHHL rabbits. All other data were examined by the ShapiroWilk test to verify normal distribution. The $t$-test was used. In all cases, a $P$ value of $<0.05$ was considered significant.

\section{Results}

\section{Influence of Long-Term Hypertension on Heart and Coronary Atherosclerosis}

Plasma levels of lipids, food intake, and body weight during the experiment were not significantly different between HTN-WHHL and NTN-WHHL rabbits (Supplemental Figures S1 and S2); however, HTN-WHHL rabbits exhibited significantly and constantly high blood pressure compared with NTN-WHHL rabbits (Figure 1). The mean systolic blood pressure of HTN-WHHL was maintained at approximately $130 \mathrm{~mm} \mathrm{Hg}$ in HTN-WHHL rabbits, whereas that of NTNWHHL rabbits was approximately $100 \mathrm{~mm} \mathrm{Hg}$ during the experiment. After becoming HTN after surgery, HTN-WHHL rabbits started to die suddenly from 34 weeks, and by 56 weeks all HTN-WHHL rabbits died. None of the NTN-WHHL rabbits died during the experiment (Figure 1). Pathologic examinations were performed, and hearts and aortas were compared between HTN- and NTN-WHHL rabbits.

\section{Myocardial Hypertrophy}

Grossly, hearts of HTN-WHHL rabbits were visibly larger in size, and the average ventricular wall thickness was greater than that in the NTN-WHHL group (Figure 2A), suggesting the presence of myocardial hypertrophy in HTNWHHL rabbits. In addition, obvious whitish scar lesions (chronic MI) were observed on the surface of hearts in five of seven HTN-WHHL rabbits $(71 \%)$, but none were noted in the NTN-WHHL rabbits.

\section{MI}

Histologic examinations confirmed that HTN-WHHL rabbits with scar lesions on the heart surface had transmural MI. MI lesions were characterized by marked fibrosis in which cardiomyocyte eosinophilic degeneration, hemorrhage, angiogenesis, and macrophage infiltration were contained (Figure 2B). MI was found in the left ventricle (6 of 7 HTN-WHHL rabbits), the right ventricle (6 of 7 HTN-WHHL rabbits), the interventricular septa (6 of 7 HTN-WHHL rabbits), and papillary myocardium (7 of 7 HTN-WHHL rabbits). Small foci of MI lesions were only found in the papillary myocardium of one NTN-WHHL rabbit. 


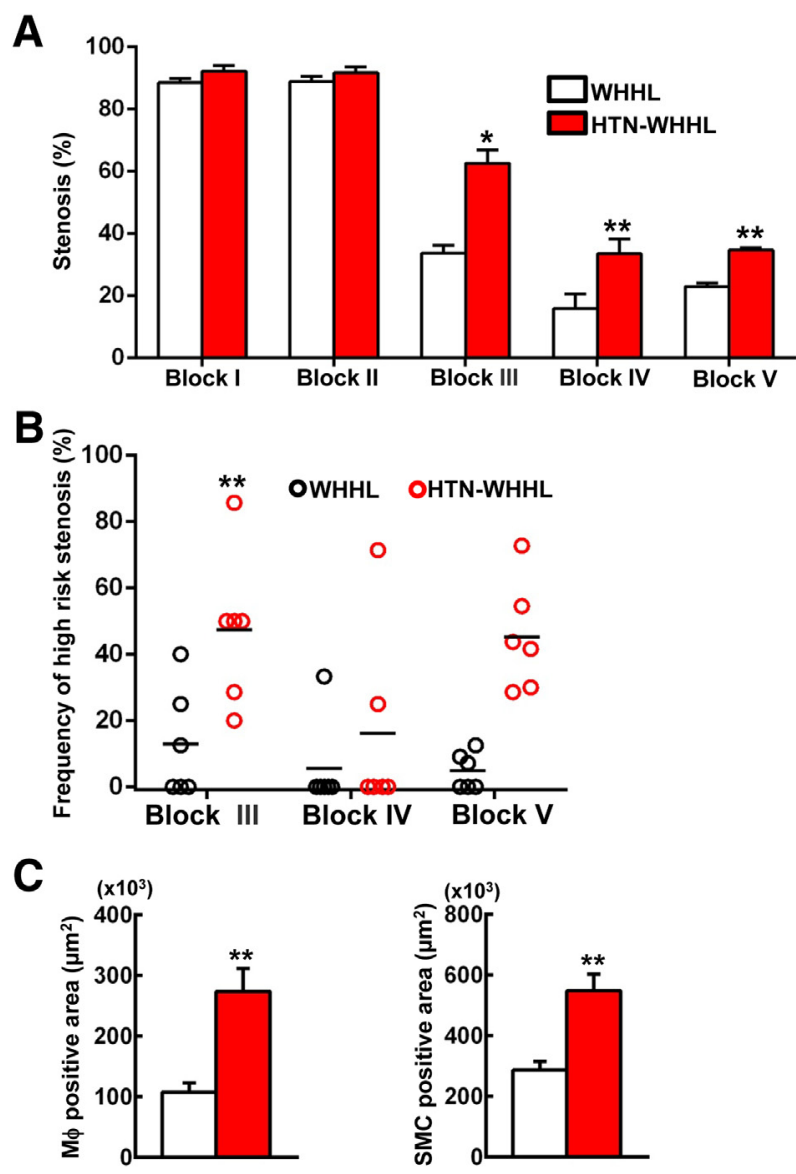

Figure 4 Quantitation of coronary stenosis in each block (A), percentage of high-risk stenosis in blocks III to V (B), and macrophages (M $\phi)$ and smooth muscle cells (SMCS) in the lesions (C). For the analysis of coronary stenosis, specimens were stained with elastica van Gieson and measured by using the described methods. In blocks III to V, there were many occlusive lesions $(>80 \%)$ defined as high-risk stenosis. High-risk stenosis (\%) in blocks III to V was calculated as the number of occlusive arteries divided by the total number of coronary arteries in each section. Immunohistochemical staining was performed by using monoclonal antibodies against either M $\phi$ or $\alpha$-smooth muscle actin for SMCs, and cellular content in all blocks was quantified with an image analysis system. Data are expressed as means \pm SEM. $n=7$ normotensive Watanabe heritable hyperlipidemic (WHHL) rabbits; $n=8$ hypertensive (HTN)-WHHL rabbits. ${ }^{*} P<0.05,{ }^{*} P<0.01$ versus normotensive WHHL rabbits.

\section{Cardiac Fibrosis}

In addition to MI areas in which diffuse fibrosis accompanied by inflammatory cell infiltration and angiogenesis was observed as described in the section above, there was prominent cardiac fibrosis in non-MI areas of HTN-WHHL rabbits. In these areas, there were increased contents of collagen between cardiomyocytes (Figure 2C). Morphometric analysis revealed that the fibrosis area (including both MI and non-MI) stained by Masson's trichrome was increased by 5-fold, 3.6-fold, and 4.6-fold in the left ventricle, the right ventricle, and the interventricular septa, respectively, of HTN-WHHL rabbits compared with NTNWHHL rabbits (Figure 2C).

\section{Coronary Atherosclerosis}

Because there was marked MI and myocardial hypertrophy in HTN-WHHL rabbits (Figure 2D), coronary atherosclerosis was next analyzed. The main trunks (epicardial coronary arteries) of the left (block I) and right (block II) coronary arteries of both groups exhibited prominent atherosclerosis with lumen stenosis $(>90 \%)$ (Figure 3A). The lesions in the main trunks were fibrotic with few macrophages and SMCs (Figure 3A). Moreover, small coronary arteries (with a diameter ranging from 200 to $500 \mu \mathrm{m})$ or arterioles $(<200 \mu \mathrm{m})$ within the myocardium contained in blocks III to $\mathrm{V}$ had marked atherosclerotic lesions (Figure 3, B and C). Compared with those of NTN-WHHL rabbits, arterioles of HTN-WHHL rabbits demonstrated two pathologic features. First was the presence of arteriolar medial hyperplasia, intimal hyperplasia, and hyalinization presumably caused by hypertension. Second, these arterioles in HTNWHHL rabbits were often complicated by atherosclerosis, and these lesions often contained both hyalinization and foam cell formation (mixed lesions) (Figure 3C). Quantification of the lumen stenosis revealed that, although the stenosis of the main trunks of the left and right coronary arteries was not significantly different between the two groups, small arteries and arterioles (blocks III to V) in HTN-WHHL rabbits had a significantly higher stenosis rate than that in NTN-WHHL rabbits (Figure 4A). Many small arteries and arterioles in HTN-WHHL rabbits were nearly or completely occluded, completely blocking coronary arterial lumens (Figures 2D and 3). By quantification of occlusive arteriolosclerosis (defined as lesions with stenosis $>80 \%$ among all arterioles that ranged from 100 to 200 $\mu \mathrm{m}$ on block V), it was found that occlusive lesions in HTNWHHL rabbits accounted for $40 \%$ (range, $30 \%$ to $73 \%$ ) of all vessels compared with 5\% (range, $0 \%$ to $12 \%$ ) in NTN-WHHL rabbits (Figure 4B). Furthermore, these coronary lesions were accompanied by an increased number of macrophages and SMCs compared with NTN-WHHL rabbits (Figure 4C).

\section{Influence of Long-Term Hypertension on Aortic Atherosclerosis}

The aortic lesions in HTN-WHHL rabbits were studied. Both HTN- and NTN-WHHL rabbits developed prominent aortic atherosclerosis, and the aortic surface was almost entirely stained by Sudan IV staining (Figure 5A). However, the lesions in the thoracic aorta (1.1-fold increase over NTN; $P<0.05)$ and abdominal aorta (1.3-fold increase over NTN; $P<0.05)$ were significantly increased in HTN-WHHL rabbits compared with NTN-WHHL rabbits Aortic lesions were fibrotic and more common on the extracellular matrix, but there were fewer macrophages and SMCs in both groups on histologic examinations (Figure 5B). Microscopically, the intimal lesions of HTN-WHHL rabbits were thick, and the microscopic lesion area was significantly increased in all parts of the aorta: 1.6-fold increase in the aortic arch, 1.4-fold increase in the thoracic aorta, and 1.4-fold increase in the abdominal aorta compared with the NTN-WHHL group. The 

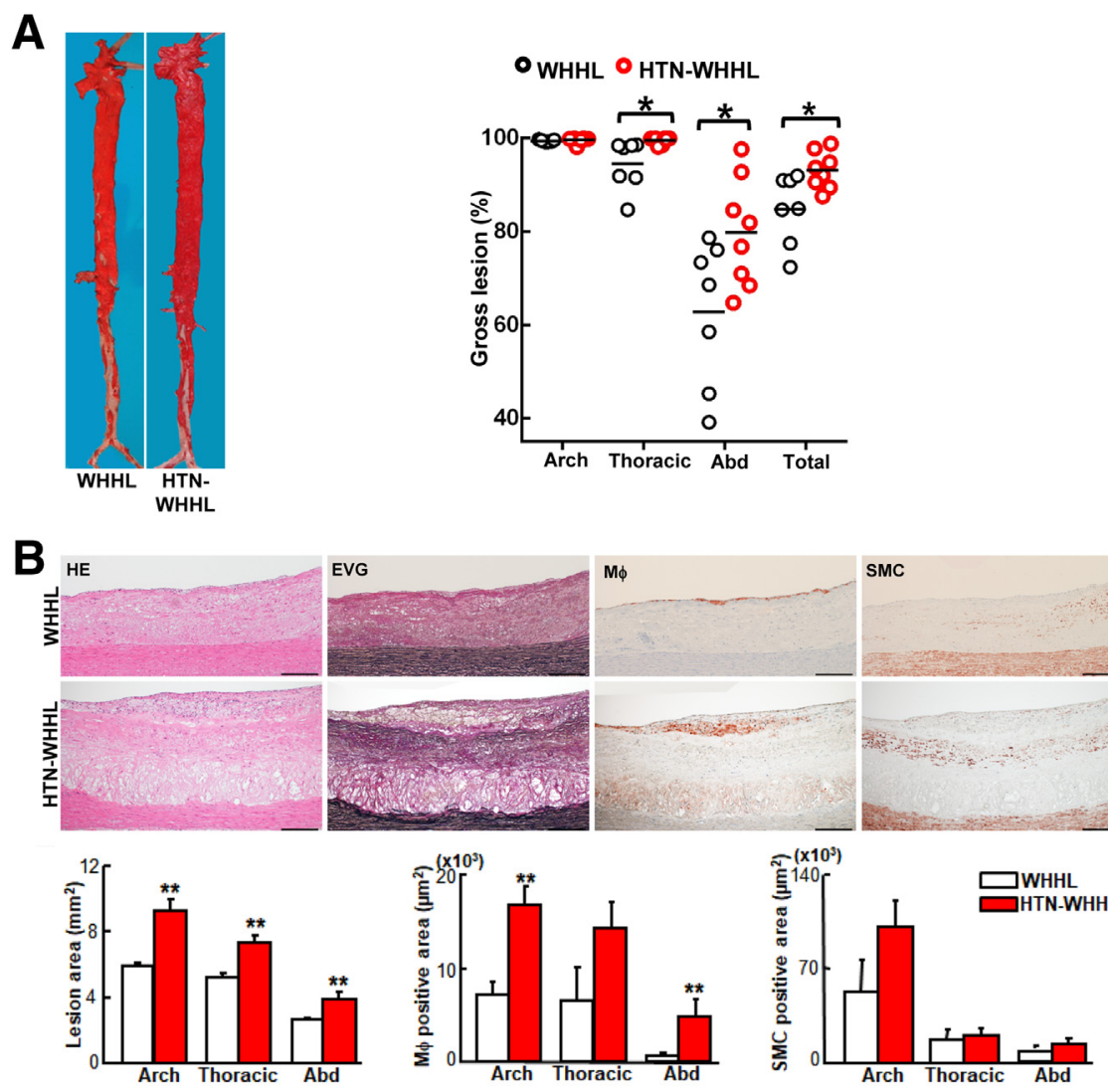

C
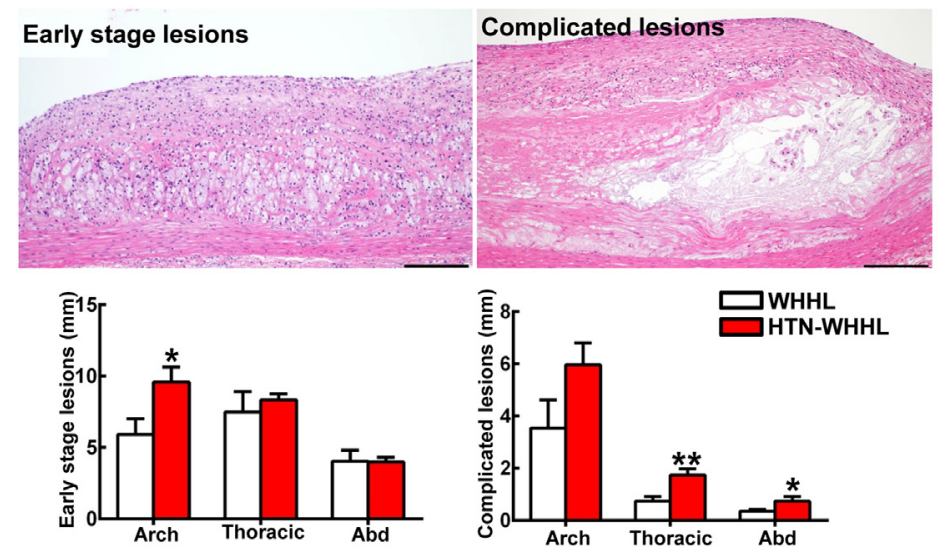

Figure 5 Analysis of gross aortic atherosclerosis. A: Representative images of aortas stained with Sudan IV. The lesion area (defined by the sudanophilic area) was quantified by using an image analysis system. Each dot represents the lesion area of an individual animal. B: Representative micrographs of the aortic arch lesions from normotensive Watanabe heritable hyperlipidemic (WHHL) and hypertensive (HTN)-WHHL rabbits. Serial sections of the aortic arch were stained with hematoxylin and eosin (HE) and elastica van Gieson (EVG) or were immunohistochemically stained with monoclonal antibodies against either macrophages (M $\phi)$ or $\alpha$-smooth muscle actin for smooth muscle cells (SMCs). Intimal lesions on EVGstained sections and positively stained areas of $M \phi$ and SMCs were quantified with an image analysis system. C: Representative images of earlystage lesions (fatty streaks) and complicated lesions or advanced lesions, that is, lesions containing a fibrotic cap and a lipid or necrotic core with or without calcification. To quantify lesion size on each section, the total length of each lesion in the aortic arch (eight specimens), thoracic aorta (20 specimens), and abdominal (Abd) aorta (30 specimens) was calculated for comparison. Data are expressed as means \pm SEM. $n=7$ normotensive WHHL rabbits; $n=8$ HTNWHHL rabbits. ${ }^{*} P<0.05,{ }^{*} P P<0.01$ versus normotensive WHHL rabbits. Scale bars $=200 \mu \mathrm{m}$. increased aortic lesion area in each part was also associated with increased macrophages in HTN-WHHL rabbits: 2.5 -fold increase $(P<0.01)$ in the aortic arch, 2.2-fold increase $(P=0.05)$ in the thoracic aorta, and 8.5 -fold increase $(P<0.01)$ in the abdominal aorta compared with the NTNWHHL group. Lesional SMCs in the HTN-WHHL group were slightly increased even though not significantly (Figure 5B).

Because the 1.7-year-old WHHL rabbits (at the time of analysis) developed severe atherosclerosis in the aorta, the proportion of early-stage lesions (fatty streaks) and complicated lesions (fibrous plaques), which contained lipid cores, calcification, and hemorrhage, were further quantified by using hematoxylin and eosin-stained specimens. Compared with NTN-WHHL rabbits, advanced lesions were increased in all parts of the aorta: 1.7-fold increase in the aortic arch, 2.4-fold increase in the thoracic aorta, and 2.1-fold increase in the abdominal aorta in HTN-WHHL rabbits, although earlystage lesions in the aortic arch (1.7-fold increase) were also increased in HTN-WHHL rabbits (Figure 5C). The fibrotic plaques were typically covered by a fibrous cap and contained lipid cores in the center, and/or were often associated with calcification, including calcium vesicle deposition, ossification, and chondrification (Figure 6A). Extensive analysis of these advanced aortic lesions in HTN-WHHL rabbits further revealed two striking histologic features. First, there was 
frequent intraplaque hemorrhage in the lesions; red blood cells were either within neoformed fatty streaks on the top of fibrous lesions, associated with neoformed capillaries, or on the shoulder of the fibrous plaques (Figure 6B). In these

A

A Fibrous plaques
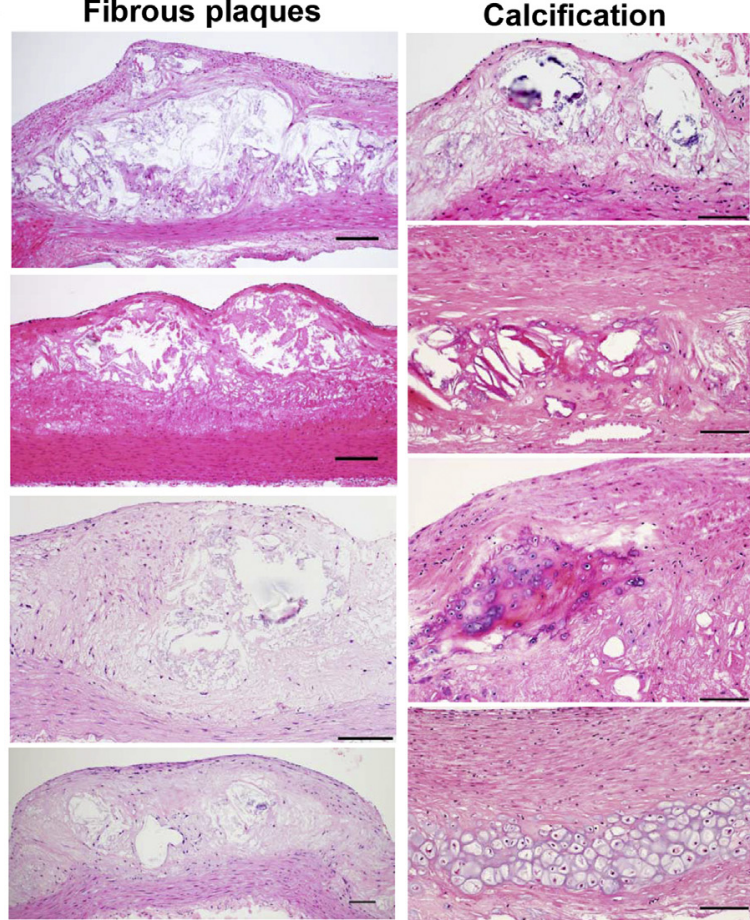

B
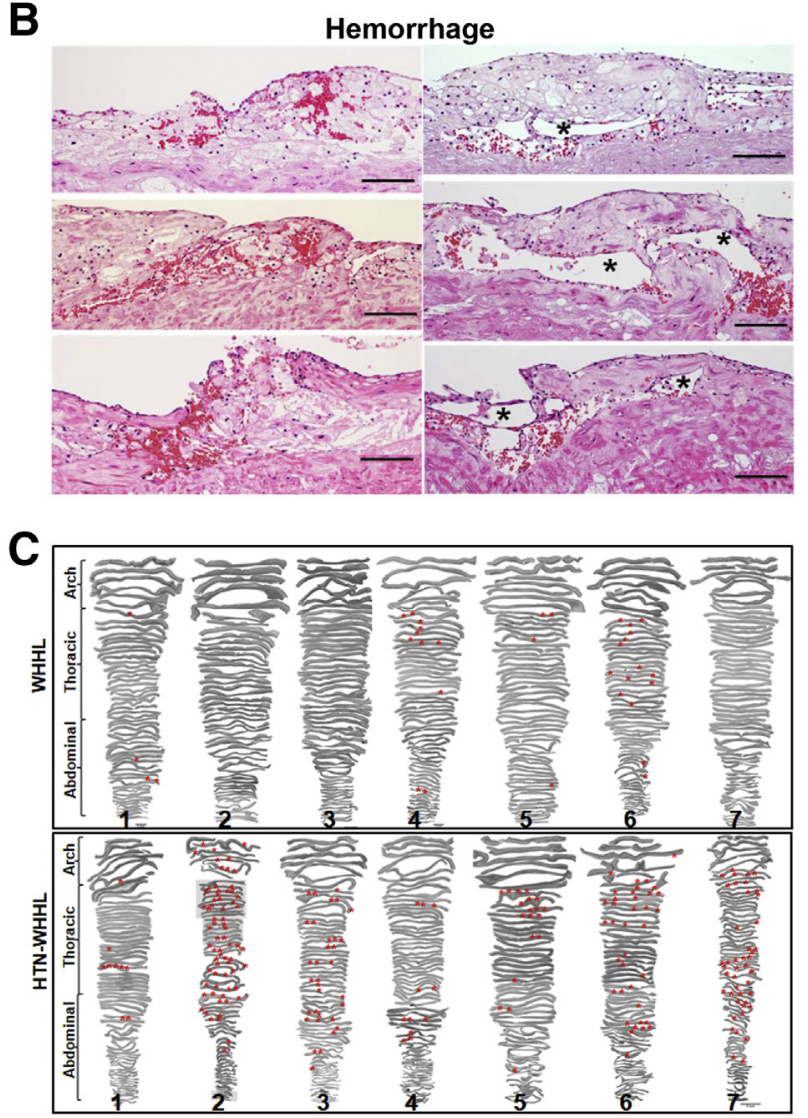

hemorrhagic areas, oxidized LDLs were also frequently colocalized with foam cells (Supplemental Figure S3).

These hemorrhagic loci were plotted on the sections (Figure 6C) and all HTN-WHHL rabbits (Note that rabbits 2, 3, 6, and 7 had extensive hemorrhage) exhibited this feature, whereas only four NTN-WHHL rabbits (rabbits 1, 4, 5, and 6) had a few hemorrhagic sites. Second, some fibrous plaques in three HTN-WHHL rabbits (rabbits 2, 6, and 7) demonstrated vulnerable histologic features associated with hemorrhage and thrombosis (Figure 7) in the vicinity of the fibrous plaques, whereas no NTN-WHHL rabbits exhibited such features. Furthermore, in vulnerable plaques with hemorrhage, there was accumulation of macrophages, which colocalized with matrix metalloproteinase (MMP)-1, MMP-9, and MMP-12 immunoreactive proteins (Figure 7).

\section{Other Pathologic Changes}

In addition to hearts and aortas as described in the section above, other organs, including the brain, lungs, kidneys, intestines, and adrenal glands, were also analyzed. Compared with NTN-WHHL rabbits, arteriolosclerosis was also found in the renal and pulmonary arterioles of HTN-WHHL rabbits (data not shown). Kidneys of HTN-WHHL rabbits showed enlarged size of glomeruli with focal tubulointerstitial fibrosis, which was not present in NTN-WHHL rabbits. Analysis of plasma markers for renal functions showed that plasma levels of creatinine but not urea were significantly increased in HTN-WHHL rabbits, suggesting that renal functions were impaired (Supplemental Figure S2). Plasma levels of angiotensin II was also significantly higher in HTNWHHL rabbits than that in NTH-WHHL rabbits (Supplemental Figure S2). Lungs of HTN-WHHL rabbits showed slight hyperemia, whereas there were not apparent differences in the brain, intestines, and adrenal glands between NTN- and HTN-WHHL rabbits.

\section{Discussion}

Although it is known that elevated blood pressure can augment the development of atherosclerosis in monkeys, ${ }^{25}$

\footnotetext{
Figure 6 Histologic analysis of aorta atherosclerotic lesions. A: Representative images of different types of advanced lesions in hypertensive (HTN) Watanabe heritable hyperlipidemic (WHHL) rabbits: various fibrous plaques containing a typical lipid or necrotic core covered by a fibrous cap and diverse calcified lesions, including calcium vesicle deposition, ossification, and chondrification. B: There are many hemorrhagic sites in the lesions of HTN-WHHL rabbits: intraplaque hemorrhage either within the neoformed fatty streaks on the top of fibrotic lesions (top left panel and middle left panel), at the shoulder area (bottom left panel), or in the vicinity of the capillaries (right column). Capillary lumens are indicated by asterisks. C: Illustration of hemorrhagic sites on the entire aorta. Hematoxylin and eosin-stained specimens were used to observe hemorrhagic foci (red asterisks) under a light microscope, and hemorrhagic foci were plotted on the sections scanned. Rabbit number is labeled at the bottom of each aorta. Scale bars $=100 \mu \mathrm{m}$.
} 

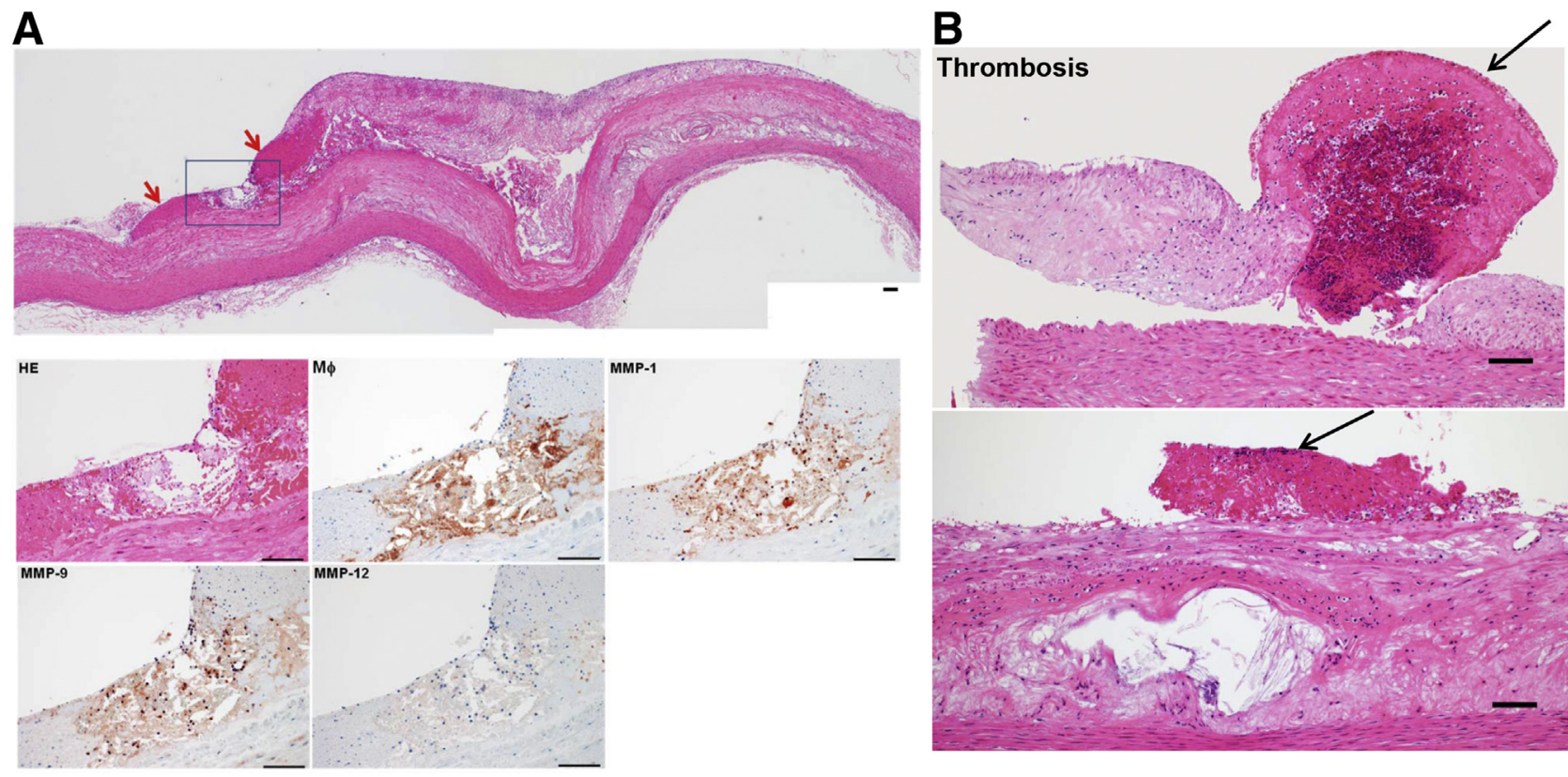

Figure 7 A: A representative vulnerable plaque of a hypertensive (HTN) Watanabe heritable hyperlipidemic (WHHL) rabbit composed of a lipid core at the center and hemorrhage at the shoulder area (red arrows). Serial paraffin sections were stained with hematoxylin and eosin (HE) or immunohistochemically stained with monoclonal antibodies against macrophages $(M \phi)$ and matrix metalloproteinase (MMP)-1, -9 , or -12 (boxed area, magnified in bottom rows). In the hemorrhagic area defined by the blue square, there was accumulation of macrophages colocalized with MMP-1, MMP-9, and MMP-12 immunoreactive proteins (bottom row). B: Thrombus formation on the aortic lesions of HTN-WHHL rabbits. In the top panel, a thrombus (arrow) was located between two fibrotic lesions in the aorta. The left lesion, seemingly separated from the aortic wall, was possibly due to an artifact during the specimen preparation. In the bottom panel, a thrombus (arrow) was located on the top of a fibrous plaque with a necrotic core at the center. Scale bars $=100 \mu \mathrm{m}$.

baboons, ${ }^{4}$ dogs, ${ }^{3}$ rats, ${ }^{26}$ and rabbits, ${ }^{5,27}$ it has not been clarified whether chronic hypertension affects cardiovascular complications such as MI. In this study, we investigated the long-term ( $>1$ year) effects of hypertension on WHHL rabbits, which develop spontaneous hypercholesterolemia and atherosclerosis on a chow diet because of the genetic deficiency of LDL receptor functions. ${ }^{28,29}$ WHHL rabbits are also known to be susceptible to coronary atherosclerosis and $\mathrm{MI},{ }^{13}$ which enabled us to examine whether prolonged hypertension affects coronary atherosclerosis and subsequent MI. Compared with NTN-WHHL rabbits, it was found that prolonged hypertension markedly influenced the cardiovascular pathologic process in HTN-WHHL rabbits. First, chronic hypertension led to enhanced aortic atherosclerosis in HTN-WHHL rabbits. The gross lesion area of aortic atherosclerosis was slightly but significantly increased in the thoracic and abdominal aortas in HTN-WHHL rabbits; however, it is possible that the aortic lesions in old WHHL rabbits ( $>1$ year old) were already saturated because of severe hypercholesterolemia. Thus, the net expansion of the lesions induced by hypertension was limited. The lesions of HTN-WHHL rabbits were characterized by more advanced lesions, including more fibrous plaques, calcification, and hemorrhage, suggesting that prolonged hypertension accelerates the progression of advanced atherosclerotic lesions. One of the most notable features of aortic lesions in HTN-WHHL rabbits was the frequent presence of intraplaque hemorrhage, which was often around macrophages, and unstable plaques that mimic human unstable lesions. ${ }^{28}$ It is well recognized that intraplaque hemorrhage is a feature of complicated and vulnerable plaques. ${ }^{30-32}$ From morphologic observations, it was concluded that hemorrhage in the plaques is either derived from the intima because red blood cells are trapped in the plaque or from neoformed capillaries because hemorrhage is in close proximity to capillaries in the plaques. It is possible that chronic hypertension impairs the integrity of vascular intima, causing erythrocytes to leak in the plaques. Of note, ox-LDL deposition was often seen in the areas with hemorrhage, suggesting that ox-LDLs may play a role in hemorrhage, although this remains to be verified in the future. In human aortic lesions, neovascular density is correlated with intraplaque hemorrhages and is considered to be involved in plaque vulnerability. ${ }^{33}$ It is currently unknown; however, whether chronic hypertension or the enhanced lesion size due to hypertension provokes hypoxic conditions in the intima milieu, thereby facilitating capillary neoformation by up-regulation of hypoxia-inducible factor $1 \alpha$ and the vascular endothelial growth factor pathway. ${ }^{34-36}$ Therefore, the fate of erythrocytes in plaques may be determined by two possible processes: phagocytosis by macrophages or hemolysis. It has been reported that erythrophagocytosis leads to iron accumulation in macrophages, which in turn destabilizes atherosclerotic plaques. ${ }^{37-39}$ If these erythrocytes trapped in the plaques undergo hemolysis, hemoglobin and erythrocyte membrane components are likely released 
into the plaque. In this situation, free hemoglobin may act as a reactive oxidative stress inducer, which enhances the burden of plaques. ${ }^{40,41}$

Because such advanced lesions in HTN-WHHL rabbits were not observed in experimental animals with a short period of hypertension in previous studies, ${ }^{5}$ it was hypothesized that hypertension duration or persistence time is another key factor for lesion nature in the setting of hypercholesterolemia. To examine this possibility, Japanese white (wild-type) rabbits with renovascular hypertension were fed with a cholesterol-rich diet for 16 weeks, and it was found that short-term exposure to hypertension led to increased aortic gross lesions by 3.5-fold (NTN rabbits $24.3 \% \pm 3.18 \%$ versus HTN rabbits $72.9 \% \pm 5.68 \%$; $P<0.01$ ), but it did not alter the nature of the lesions such as hemorrhage shown in the present study. Hypertension may affect the pathophysiological functions of the cardiovascular system through multiple mechanisms. ${ }^{42}$ First, elevated blood pressure can generate direct mechanical force, pressure, and shear stress to vascular endothelial cells to cause endothelial injury. Second, hypertension can enhance atherogenic lipoprotein entry into the intima. Third, hypertension can increase the adherence of monocytes to endothelial cells and emigration into the subintima. In addition, the renin-angiotensin system, a key mediator of blood pressure, plays a critical role in cardiovascular homeostasis. According to our previous study, infusion of angiotensin II can destabilize coronary plaques and trigger the formation of thrombosis in WHHL rabbits. ${ }^{16}$ In the present study, the $1 \mathrm{~K} 1 \mathrm{C}$ rabbit model was used in which hypertension was induced by both the renin-angiotensin system and the enhancement of cardiac sympathetic activity. ${ }^{43-45}$ In the unstable plaques, there is focal accumulation of macrophages colocalized with different MMPs, suggesting that up-regulation of MMPs induced by hypertension may be involved in the formation of advanced lesions. However, no rupture or aneurysms were observed in the aorta, as seen in apolipoprotein E knockout mice induced by angiotensin II infusion, ${ }^{8}$ suggesting that some other factors are required to trigger plaque rupture even though both hypercholesterolemia and hypertension are present in these animals.

One notable observation was that HTN-WHHL rabbits died from 34 weeks, whereas no NTN-WHHL rabbits died. The death of HTN-WHHL rabbits was likely due to either MI or heart failure or both. This assumption is supported by these rabbits suddenly dying without any antecedent signs such as inactivity, less intake of food, or paralysis. Second, pathologic examinations revealed that all HTN-WHHL rabbits had marked MI and myocardial hypertrophy, which was not evident in NTN-WHHL rabbits. It is well known that hypertension can increase the myocardial load, enhance cardiac fibrosis, and eventually cause myocardial hypertrophy. ${ }^{44,46,47}$ Furthermore, there was severe coronary atherosclerosis and hyaline arteriolosclerosis in HTNWHHL rabbits, which may also cause cardiac dysfunction or cardiac death. Note that hyaline arteriolosclerosis caused by hypertension was often complicated by fatty streaks in the small arteries of HTN-WHHL rabbits. Therefore, in the setting of hypercholesterolemia, hypertension may synergistically mediate lesion formation and progression even in small arteries. Although hypertension may play a major role in the death of $1 \mathrm{~K} 1 \mathrm{C}$-induced HTN-WHHL rabbits, other factors such as renal or pulmonary dysfunctions may be also involved. Moreover, these HTN-WHHL rabbits also showed increased plasma levels of creatinine and angiotensin II (Supplemental Figure S4), even though angiotensin II levels were much lower than angiotensin II-infused WHHL rabbits in which angiotensin II levels were approximately 300 to $500 \mathrm{pg} / \mathrm{mL}^{16}$ Therefore, future investigations are required to elucidate their relationships or interactions.

The present study provided several important implications. First, it is clear that long-term hypertension enhances the development of advanced atherosclerosis; therefore, effective control of high blood pressure is expected to reduce the progression of atherosclerosis. Second, the observation that prolonged hypertension can lead to a high prevalence of cardiac death further suggests that therapeutic control of high blood pressure may reduce the risk of cardiovascular events in hypercholesterolemia patients with hypertension.

\section{Conclusion}

The present study demonstrates that prolonged hypertension induces intraplaque hemorrhage, plaque vulnerability, and increases the prevalence of MI and mortality in HTNWHHL rabbits. Effective management of high blood pressure may prevent cardiovascular events in HTN patients. Advanced lesions of atherosclerosis observed in HTNWHHL rabbits may provide opportunities to investigate the molecular mechanisms of plaque vulnerability and rupture induced by hypertension.

\section{Acknowledgments}

We thank Zhengchao Liu and Dedong Kang for their help with rabbit surgery.

Y.E.C. and J.F. designed experiments and analyzed and interpreted data; B.N., Y.C., A.B.W., H.Y., J.Z., Y.W., and J.L. performed experiments and analyzed the data; M.S. provided Watanabe heritable hyperlipidemic rabbits; H.I. provided antibodies; Y.C., A.B.W., Y.E.C., and J.F. wrote the manuscript.

\section{Supplemental Data}

Supplemental material for this article can be found at https://doi.org/10.1016/j.ajpath.2018.08.007. 


\section{References}

1. Solberg LA, Strong JP: Risk factors and atherosclerotic lesions. A review of autopsy studies. Arteriosclerosis 1983, 3:187-198

2. Robertson WB, Strong JP: Atherosclerosis in persons with hypertension and diabetes mellitus. Lab Invest 1968, 18:538-551

3. Moses C: Development of atherosclerosis in dogs with hypercholesterolemia and chronic hypertension. Circ Res 1954, 2:243-247

4. McGill HC Jr, Carey KD, McMahan CA, Marinez YN, Cooper TE, Mott GE, Schwartz CJ: Effects of two forms of hypertension on atherosclerosis in the hyperlipidemic baboon. Arteriosclerosis 1985, 5:481-493

5. Chobanian AV, Lichtenstein AH, Nilakhe V, Haudenschild CC, Drago R, Nickerson C: Influence of hypertension on aortic atherosclerosis in the Watanabe rabbit. Hypertension 1989, 14:203-209

6. Julius S, Kjeldsen SE, Weber M, Brunner HR, Ekman S, Hansson L, Hua TS, Laragh J, McInnes GT, Mitchell L, Plat F, Schork A, Smith B, Zanchetti A; VALUE Trial Group: Outcomes in hypertensive patients at high cardiovascular risk treated with regimens based on valsartan or amlodipine: the VALUE randomised trial. Lancet 2004, 363: 2022-2031

7. Chobanian AV: Effects of hypertension on arterial gene expression and atherosclerosis. Adv Exp Med Biol 1991, 308:45-53

8. Daugherty A, Manning MW, Cassis LA: Angiotensin II promotes atherosclerotic lesions and aneurysms in apolipoprotein E-deficient mice. J Clin Invest 2000, 105:1605-1612

9. Weiss D, Kools JJ, Taylor WR: Angiotensin II-induced hypertension accelerates the development of atherosclerosis in apoE-deficient mice. Circulation 2001, 103:448-454

10. Sugiyama F, Haraoka S, Watanabe T, Shiota N, Taniguchi K, Ueno Y, Tanimoto K, Murakami K, Fukamizu A, Yagami K: Acceleration of atherosclerotic lesions in transgenic mice with hypertension by the activated renin-angiotensin system. Lab Invest 1997, 76:835-842

11. Chobanian AV: The influence of hypertension and other hemodynamic factors in atherogenesis. Prog Cardiovasc Dis 1983, 26:177-196

12. Shiomi M, Fan J: Unstable coronary plaques and cardiac events in myocardial infarction-prone Watanabe heritable hyperlipidemic rabbits: questions and quandaries. Curr Opin Lipidol 2008, 19:631-636

13. Shiomi M, Ito T, Yamada S, Kawashima S, Fan J: Development of an animal model for spontaneous myocardial infarction (WHHLMI rabbits). Arterioscler Thromb Vasc Biol 2003, 23:1239-1244

14. Nickerson CJ, Haudenschild CC, Chobanian AV: Effects of hypertension and hyperlipidemia on the myocardium and coronary vasculature of the WHHL rabbit. Exp Mol Pathol 1992, 56:173-185

15. Chobanian AV, Haudenschild CC, Nickerson C, Drago R: Antiatherogenic effect of captopril in the Watanabe heritable hyperlipidemic rabbit. Hypertension 1990, 15:327-331

16. Li S, Wang YN, Niimi M, Ning B, Chen Y, Kang D, Wang Z, Yu Q, Waqar AB, Liu E, Zhang J, Shiomi M, Chen YE, Fan J: Angiotensin II destabilizes coronary plaques in Watanabe heritable hyperlipidemic rabbits. Arterioscler Thromb Vasc Biol 2016, 36:810-816

17. Committee for the Update of the Guide for the Care and Use of Laboratory Animals; National Research Council: Guide for the Care and Use of Laboratory Animals: Eighth Edition. Washington, DC, National Academies Press, 2011

18. Waqar AB, Koike T, Yu Y, Inoue T, Aoki T, Liu E, Fan J: High-fat diet without excess calories induces metabolic disorders and enhances atherosclerosis in rabbits. Atherosclerosis 2010, 213:148-155

19. Wang C, Nishijima K, Kitajima S, Niimi M, Yan H, Chen Y, Ning B, Matsuhisa F, Liu E, Zhang J, Chen YE, Fan J: Increased hepatic expression of endothelial lipase inhibits cholesterol diet-induced hypercholesterolemia and atherosclerosis in transgenic rabbits. Arterioscler Thromb Vasc Biol 2017, 37:1282-1289

20. Koike $T$, Liang J, Wang $X$, Ichikawa $T$, Shiomi $M$, Sun $H$, Watanabe T, Liu G, Fan J: Enhanced aortic atherosclerosis in transgenic Watanabe heritable hyperlipidemic rabbits expressing lipoprotein lipase. Cardiovasc Res 2005, 65:524-534
21. Li S, Liang J, Niimi M, Bilal Waqar A, Kang D, Koike T, Wang Y, Shiomi M, Fan J: Probucol suppresses macrophage infiltration and MMP expression in atherosclerotic plaques of WHHL rabbits. J Atheroscler Thromb 2014, 21:648-658

22. Stary HC, Chandler AB, Dinsmore RE, Fuster V, Glagov S, Insull W Jr, Rosenfeld ME, Schwartz CJ, Wagner WD, Wissler RW: A definition of advanced types of atherosclerotic lesions and a histological classification of atherosclerosis. A report from the Committee on Vascular Lesions of the Council on Arteriosclerosis, American Heart Association. Circulation 1995, 92:1355-1374

23. Yamada S, Wang KY, Tanimoto A, Fan J, Shimajiri S, Kitajima S, Morimoto M, Tsutsui M, Watanabe T, Yasumoto K, Sasaguri Y: Matrix metalloproteinase 12 accelerates the initiation of atherosclerosis and stimulates the progression of fatty streaks to fibrous plaques in transgenic rabbits. Am J Pathol 2008, 172:1419-1429

24. Fan J, Kitajima S, Watanabe T, Xu J, Zhang J, Liu E, Chen YE: Rabbit models for the study of human atherosclerosis: from pathophysiological mechanisms to translational medicine. Pharmacol Ther 2015, 146: 104-119

25. Pick R, Johnson PJ, Glick G: Deleterious effects of hypertension on the development of aortic and coronary atherosclerosis in stumptail macaques (Macaca speciosa) on an atherogenic diet. Circ Res 1974, 35: $472-482$

26. Koletsky S, Roland C, Rivera-Velez JM: Rapid acceleration of atherosclerosis in hypertensive rats on high fat diet. Exp Mol Pathol 1968, 9:322-338

27. Heptinstall RH, Barkley H, Porter KA: The relative roles of blood cholesterol level and blood pressure level in the production of experimental aortic atheroma in rabbits. Angiology 1958, 9:84-87

28. Watanabe Y: Serial inbreeding of rabbits with hereditary hyperlipidemia (WHHL-rabbit). Atherosclerosis 1980, 36:261-268

29. Yamamoto T, Bishop RW, Brown MS, Goldstein JL, Russell DW: Deletion in cysteine-rich region of LDL receptor impedes transport to cell surface in WHHL rabbit. Science 1986, 232:1230-1237

30. Takaya N, Yuan C, Chu BC, Saam T, Polissar NL, Jarvik GP, Isaac C, McDonough J, Natiello C, Small R, Ferguson MS, Hatsukami TS: Presence of intraplaque hemorrhage stimulates progression of carotid atherosclerotic plaques: a high-resolution magnetic resonance Imaging study. Circulation 2005, 111:2768-2775

31. Fleiner M, Kummer M, Mirlacher M, Sauter G, Cathomas G, Krapf R, Biedermann BC: Arterial neovascularization and inflammation in vulnerable patients: early and late signs of symptomatic atherosclerosis. Circulation 2004, 110:2843-2850

32. Kolodgie FD, Gold HK, Burke AP, Fowler DR, Kruth HS, Weber DK, Farb A, Guerrero LJ, Hayase M, Kutys R, Narula J, Finn AV, Virmani R: Intraplaque hemorrhage and progression of coronary atheroma. N Engl J Med 2003, 349:2316-2325

33. Moreno PR, Purushothaman R, Fuster V, Echeverri D, Truszczynska H, Sharma SK, Badimon JJ, O'Connor WN: Plaque neovascularization is increased in ruptured atherosclerotic lesions of human aorta: implications for plaque vulnerability. Circulation 2004, 110:2032-2038

34. Pugh CW, Ratcliffe PJ: Regulation of angiogenesis by hypoxia: role of the HIF system. Nat Med 2003, 9:677-684

35. Moreno PR, Fuster V: New aspects in the pathogenesis of diabetic atherothrombosis. J Am Coll Cardiol 2004, 44:2293-2300

36. Silvestre JS, MallatZ, Tamarat R, Duriez M, Tedgui A, Levy BI: Regulation of matrix metalloproteinase activity in ischemic tissue by interleukin-10: role in ischemia-induced angiogenesis. Circ Res 2001, 89:259-264

37. Levy AP, Moreno PR: Intraplaque hemorrhage. Curr Mol Med 2006, 6:479-488

38. Li JJ, Meng X, Si HP, Zhang C, Lv HX, Zhao YX, Yang JM, Dong M, Zhang K, Liu SX, Zhao XQ, Gao F, Liu XL, Cui TX, Zhang Y: Hepcidin destabilizes atherosclerotic plaque via overactivating macrophages after erythrophagocytosis. Arterioscler Thromb Vasc Biol 2012, 32:1158-1166 
39. Tziakas DN, Chalikias GK, Stakos D, Boudoulas H: The role of red blood cells in the progression and instability of atherosclerotic plaque. Int J Cardiol 2010, 142:2-7

40. Purushothaman M, Krishnan P, Purushothaman KR, Baber U, Tarricone A, Perez JS, Wiley J, Kini A, Sharma SK, Fuster V, Moreno PR: Genotype-dependent impairment of hemoglobin clearance increases oxidative and inflammatory response in human diabetic atherosclerosis. Arterioscler Thromb Vasc Biol 2012, 32: 2769-2775

41. Nagy E, Eaton JW, Jeney V, Soares MP, Varga Z, Galajda Z, Szentmikloski J, Mehes G, Csonka T, Smith A, Vercellotti GM, Balla G, Balla J: Red cells, hemoglobin, heme, iron, and atherogenesis. Arterioscler Thromb Vasc Biol 2010, 30:1347-1353

42. Chobanian AV, Alexander RW: Exacerbation of atherosclerosis by hypertension. Potential mechanisms and clinical implications. Arch Intern Med 1996, 156:1952-1956

43. Nussberger J, Aubert JF, Bouzourene K, Pellegrin M, Hayoz D, Mazzolai L: Renin inhibition by aliskiren prevents atherosclerosis progression: comparison with irbesartan, atenolol, and amlodipine. Hypertension 2008, 51:1306-1311

44. Borges GR, Salgado HC, Silva CA, Rossi MA, Prado CM, Fazan R Jr: Changes in hemodynamic and neurohumoral control cause cardiac damage in one-kidney, one-clip hypertensive mice. Am J Physiol Regul Integr Comp Physiol 2008, 295:R1904-R1913

45. Mazzolai L, Duchosal MA, Korber M, Bouzourene K, Aubert JF, Hao H, Vallet V, Brunner HR, Nussberger J, Gabbiani G, Hayoz D: Endogenous angiotensin II induces atherosclerotic plaque vulnerability and elicits a Th1 response in ApoE(-/-) mice. Hypertension 2004, 44:277-282

46. Lau DH, Mackenzie L, Rajendram A, Psaltis PJ, Kelly DR, Spyropoulos P, Zhang Y, Olakkengil SA, Russell CH, Brooks AG, Faull RJ, Saint DA, Kelly DJ, Rao MM, Worthley SG, Sanders P: Characterization of cardiac remodeling in a large animal "one-kidney, one-clip" hypertensive model. Blood Press 2010, 19:119-125

47. Thomas L, Gasser B, Bousquet P, Monassier L: Hemodynamic and cardiac anti-hypertrophic actions of clonidine in Goldblatt one-kidney, one-clip rats. J Cardiovasc Pharm 2003, 41:203-209 